\title{
Anti-GITR Monoclonal Antibody GWN 323
}

National Cancer Institute

\section{Source}

National Cancer Institute. Anti-GITR Monoclonal Antibody GWN 323. NCI Thesaurus.

Code $\mathrm{C128028.}$

An anti-human glucocorticoid-induced tumor necrosis factor receptor (tumor necrosis factor superfamily, member 18; TNFRSF18; GITR; CD357) agonistic monoclonal antibody, with potential immune checkpoint modulating activity. Anti-GITR antibody GWN 323 binds to and activates GITRs found on multiple types of T-cells. This stimulates the immune system, induces both the activation and proliferation of tumor-antigen-specific T effector cells (T eff), and suppresses the function of activated T regulatory cells (Tregs). This leads to tumor cell eradication. GITR, a member of the TNF receptor superfamily and T-cell receptor co-stimulator, is expressed on the surface of multiple immune cell types, including Tregs, Teffs, B-cells, and natural killer (NK) cells. Inappropriately activated T regs suppress both Teffs and T-cell receptor (TCR) signaling. 